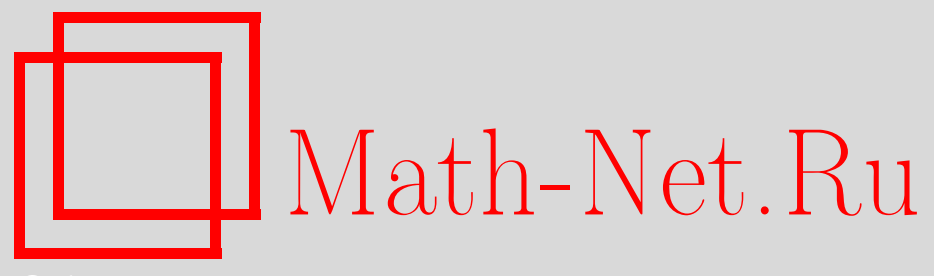

Ю. В. Малыхин, Верхние оценки погрешности оценщиков в задаче непараметрической регрессии: адаптивный случай и случай неизвестной меры $\rho_{X}$, Матем. заметкu, 2009, том 86, выпуск 5, 725-732

DOI: https://doi.org/10.4213/mzm6571

Использование Общероссийского математического портала Math-Net.Ru подразумевает, что вы прочитали и согласны с пользовательским соглашением http://www.mathnet.ru/rus/agreement

Параметры загрузки:

IP: 18.234 .197 .8

26 апреля 2023 г., $15: 27: 36$

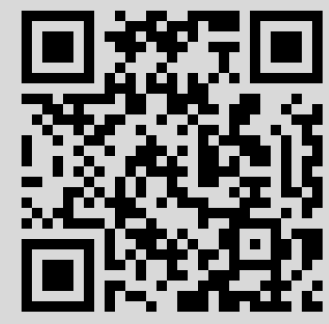


Том 86 выпуск 5 ноябрь 2009

УДК 519.234

\section{Верхние оценки погрешности оценщиков в задаче непараметрической регрессии: адаптивный случай и случай неизвестной меры $\rho_{X}$}

\section{Ю. В. Малыхин}

В работе строятся оценщики функций регрессии и доказываются теоремы об их погрешности в двух различных ситуациях.

В первом случае рассматриваются так называемые адаптивные оценщики, погрешность которых близка к оптимальной для целого семейства классов возможных функций регрессии; адаптивность оценщиков заключается в том, что они строятся без информации о выборе класса.

Во втором случае класс возможных функций регрессии фиксирован, однако маргинальная мера неизвестна, оценщик строится без какой-либо информации об этой мере. Его погрешность оказывается близкой к минимальной возможной (в наихудшем случае) погрешности.

Библиография: 7 названий.

1. Введение. В данной работе рассматривается задача регрессии. Основой для наших исследований являются статьи [1]-[4].

Пусть $X=\mathbb{R}^{d}, Y=[-M, M], \rho-$ неизвестная нам борелевская вероятностная мера на $X \times Y, \rho_{X}$ суть проекция меры $\rho$ на $X$ (т.е. $\left.\rho_{X}(S)=\rho(S \times Y), S \subset X\right)$.

Задача состоит в том, чтобы по выборке $\mathbf{z}=\mathbf{z}^{(m)}=\left(\left(x_{1}, y_{1}\right), \ldots,\left(x_{m}, y_{m}\right)\right)$, coстоящей из независимых векторов $z_{i}=\left(x_{i}, y_{i}\right) \in X \times Y$, распределенных по мере $\rho$, построить функцию $f_{\mathbf{z}}: X \rightarrow Y$, аппроксимирующую функцию регрессии

$$
f_{\rho}(x)=\int_{Y} y d \rho(y \mid x) .
$$

Отображение $\mathbf{z} \mapsto f_{\mathbf{z}}$ называется оценщиком.

Функция регрессии минимизирует величину ошибки

$$
\mathscr{E}(f)=\int_{X \times Y}(f(x)-y)^{2} d \rho
$$

по всевозможным $f$ - это следует из равенства $\mathscr{E}(f)-\mathscr{E}\left(f_{\rho}\right)=\left\|f-f_{\rho}\right\|_{L_{2}\left(\rho_{X}\right)}^{2}$. Таким образом, аппроксимация $f_{\rho}$ в метрике $L_{2}\left(\rho_{X}\right)$ равносильна минимизации функционала $\mathscr{E}(\cdot)$.

Работа выполнена при поддержке Российского фонда фундаментальных исследований (грант № 08-01-00208) и программы "Ведущие научные школы" (грант № НШ-3810.2008.1).

(C) Ю. В. МАлыхин, 2009 
Качество оценщика можно измерять вероятностью того, что он даст погрешность, большую некоторого $\varepsilon>0$ :

$$
\rho^{m}\left\{\mathbf{z}: \mathscr{E}\left(f_{\mathbf{z}}\right)-\mathscr{E}\left(f_{\rho}\right)>\varepsilon\right\} .
$$

Поскольку, вообще говоря, множество $\left\{\mathbf{z}: \mathscr{E}\left(f_{\mathbf{z}}\right)-\mathscr{E}\left(f_{\rho}\right)>\varepsilon\right\}$ может оказаться неизмеримым, под вероятностью понимается внешняя вероятность.

Разумеется, чтобы получить нетривиальные оценки таких вероятностей, нужно наложить на $\rho$ определенные ограничения. Для примера приведем теорему из [3].

Нам потребуются следующие обозначения: для множества $A$ в метрическом пространстве $S$ и $\varepsilon>0$ через $N_{\varepsilon}(A, S)$ будем обозначать количество элементов в минимальной $\varepsilon$-сети для $A$ в $S$; через $\varepsilon_{n}(A, S)$ обозначим энтропийный поперечник

$$
\varepsilon_{n}(A, S):=\inf \left\{\varepsilon>0: \log N_{\varepsilon}(A, S) \leqslant n\right\} .
$$

(Для определенности, $\log t=\log _{e} t$.)

Tеорема А [3; теорема 1.3]. Пусть класс $\Theta$ функиий из $X$ в $Y$ и мера $\mu$ на $X$ mаковы, что

$$
\varepsilon_{n}\left(\Theta, L_{2}(\mu)\right) \leqslant D n^{-r}, \quad n=1,2, \ldots .
$$

Тогда существует такой оценщик $f_{\mathbf{z}}$, что для любых $m \geqslant 240(M / D)^{2}, \varepsilon>$ $c(M, D, r) \cdot m^{-2 r /(1+2 r)}$, и любой меры $\rho$ такой, что $f_{\rho} \in \Theta, \rho_{X}=\mu$, справедлива оценка

$$
\rho^{m}\left\{\mathbf{z}: \mathscr{E}\left(f_{\mathbf{z}}\right)-\mathscr{E}\left(f_{\rho}\right) \geqslant \varepsilon\right\} \leqslant \exp \left(-\frac{m \varepsilon}{800 M^{2}}\right) .
$$

Отметим, что показатель $2 r /(1+2 r)$ нельзя увеличить; это следует из нижних оценок [2]; см. также [3].

В первой части работы мы доказываем основную лемму, из которой легко выводится теорема А. Лемма фактически доказывается в [3], но нам будет удобно выделить эти соображения в виде отдельного утверждения.

С помощью основной леммы мы получим общую теорему об адаптивных оценщиках. Не приводя здесь формулировки этой теоремы, поясним термин "адаптивный" и выпишем одно ее следствие.

Пусть заданы произвольное множество индексов $R$ и $\left\{\mathscr{M}_{r}\right\}$ - семейство классов мер на $X \times Y$. Предположим, что мера $\rho$ принадлежит некоторому классу $\mathscr{M}_{r_{0}}$, $r_{0} \in R$, но $r_{0}$ неизвестно. Требуется на основе выборки $\mathbf{z}$ построить такой адаптивный оценщик $f_{\mathbf{z}}$, что погрешность $\mathscr{E}\left(f_{\mathbf{z}}\right)-\mathscr{E}\left(f_{\rho}\right)$ была бы близка к оптимальной для класса $\mathscr{M}_{r_{0}}$. Адаптивность оценщика заключается в том, что он строится без информации об $r_{0}$. В качестве примера приведем следующую теорему.

Теорема В [4; теорема 1]. Для произволъных $0<\alpha<\beta<\infty$ рассмотрим семейство классов функиий $\left\{W_{r}\right\}, r \in[\alpha, \beta], u$ меру $\mu$, удовлетворяющие следующим условиям:

$$
\begin{gathered}
\varepsilon_{n}\left(W_{r}, L_{2}(\mu)\right) \leqslant D n^{-r}, \\
W_{r_{1}} \supset W_{r_{2}}, \quad \alpha \leqslant r_{1} \leqslant r_{2} \leqslant \beta .
\end{gathered}
$$

Существует такой оценщик $f_{\mathbf{z}}$ и константы $C_{i}=C_{i}(\alpha, \beta, D, M)>0, i=1,2$, $A_{0}=A_{0}(\alpha, \beta, D, M)>0$, что для любой мерь $\rho$ из равенства $\rho_{X}=\mu$ и включения 
$f_{\rho} \in W_{r_{0}}, r_{0} \in[\alpha, \beta]$, следует неравенство

$$
\rho^{m}\left\{\mathbf{z}: \mathscr{E}\left(f_{\mathbf{z}}\right)-\mathscr{E}\left(f_{\rho}\right) \geqslant C_{1} A m^{-2 r_{0} /\left(1+2 r_{0}\right)}\right\} \leqslant \exp \left(-C_{2} A m^{1 /(1+2 \beta)}\right)
$$

с произвольным $A>A_{0}$.

Теорему В можно вывести из нашей “общей” теоремы 1 (как и "общую" теорему 2 [4], из которой выводилась теорема В).

В следующей части работы рассматривается случай, когда мера $\rho_{X}$ неизвестна. Если оценки в случае фиксированной $\rho_{X}=\mu$ проводились в терминах энтропии в пространстве $L_{2}(\mu)$, то теперь ключевой характеристикой будет следующая величина.

ОПРЕДЕЛЕНИЕ. Комбинаторной размерностъю $\operatorname{vc}(\Theta, \varepsilon)$ класса $\Theta$ называется максимальное натуральное число $n$ такое, что существуют точки $x_{1}, \ldots, x_{n} \in X$ и значения $y_{1}, \ldots, y_{n} \in \mathbb{R}$, удовлетворяющие условию: для любого набора знаков $\sigma_{1}, \ldots, \sigma_{n} \in\{-1,1\}$ найдется функция $f \in \Theta$ такая, что

$$
\sigma_{i}\left(f\left(x_{i}\right)-y_{i}\right) \geqslant \varepsilon, \quad i=1, \ldots, n .
$$

Если таких $n$ бесконечно много, то $\operatorname{vc}(\Theta, \varepsilon)=\infty$.

В теореме 2 мы покажем, что в случае, когда мера $\rho_{X}$ неизвестна и на меру $\rho$ накладывается единственное условие $f_{\rho} \in \Theta$, существует оценщик, для которого верно неравенство

$$
\rho^{m}\left\{\mathbf{z}: \mathscr{E}\left(f_{\mathbf{z}}\right)-\mathscr{E}\left(f_{\rho}\right)>\varepsilon\right\} \leqslant c_{1} \exp \left(c_{2} \mathrm{vc}\left(\Theta, c_{3} \sqrt{\varepsilon}\right) \log \left(\frac{2}{\varepsilon}\right)-c_{4} m \varepsilon\right) .
$$

Точная формулировка теоремы и способ построения оценщика приведены далее.

2. Основная лемма. Пусть $\mathscr{H}$ - некоторый класс функций из $X$ в $Y$. Введем следующие обозначения:

$$
\mathscr{E}_{\mathbf{z}}(f):=\frac{1}{m} \sum_{i=1}^{m}\left(f\left(x_{i}\right)-y_{i}\right)^{2}, \quad f_{\mathbf{z}, \mathscr{H}}:=\arg \min _{f \in \mathscr{H}} \mathscr{E}_{\mathbf{z}}(f) .
$$

Если минимум $\mathscr{E}_{\mathbf{z}}(f)$ достигается сразу на нескольких функциях $f \in \mathscr{H}$, то мы выбираем произвольную из них.

Лемма 1. Пусть $\mathscr{N}-$ конечный набор функций $X \rightarrow Y, \# \mathscr{N}=N$. Для всякого $\delta \geqslant \min _{f \in \mathscr{N}} \mathscr{E}(f)-\mathscr{E}\left(f_{\rho}\right)$ выполняется неравенство

$$
\rho^{m}\left\{\mathbf{z}: \mathscr{E}\left(f_{\mathbf{z}, \mathscr{N}}\right)-\mathscr{E}\left(f_{\rho}\right) \geqslant 5 \delta\right\} \leqslant(N+1) \exp \left(-\frac{m \delta}{40 M^{2}}\right) .
$$

Для полноты изложения приведем полное доказательство леммы. Нам понадобится лишь неравенство Бернштейна. Оно утверждает, что для независимых, одинаково распределенных величин $\xi_{i},\left|\xi_{i}\right| \leqslant M$, выполняется неравенство

$$
\mathrm{P}\left\{\frac{1}{n} \sum_{i=1}^{n} \xi_{i}-\mathrm{E} \xi \geqslant t\right\} \leqslant \exp \left(-\frac{n t^{2}}{2(\mathrm{D} \xi+M t / 3)}\right) .
$$

Та же оценка имеет место для вероятности события $\left\{\mathrm{E} \xi-(1 / n) \sum_{i=1}^{n} \xi_{i} \geqslant t\right\}$. 
ДоКАЗАТЕЛЬСТво ЛЕммы 1. Введем обозначения

$$
\mathscr{E}_{\rho}(f):=\mathscr{E}(f)-\mathscr{E}\left(f_{\rho}\right), \quad \mathscr{E}_{\rho, \mathbf{z}}(f):=\mathscr{E}_{\mathbf{z}}(f)-\mathscr{E}_{\mathbf{z}}\left(f_{\rho}\right)
$$

Покажем, что для любой функции $f: X \rightarrow Y$ справедливы неравенства

$$
\begin{gathered}
\rho^{m}\left\{\mathbf{z}: \mathscr{E}_{\rho}(f)-\mathscr{E}_{\rho, \mathbf{z}}(f) \geqslant \frac{1}{2}\left(\mathscr{E}_{\rho}(f)+\delta\right)\right\} \leqslant \exp \left(-\frac{m \delta}{40 M^{2}}\right), \\
\rho^{m}\left\{\mathbf{z}: \mathscr{E}_{\rho}(f)-\mathscr{E}_{\rho, \mathbf{z}}(f) \leqslant-\frac{1}{2}\left(\mathscr{E}_{\rho}(f)+\delta\right)\right\} \leqslant \exp \left(-\frac{m \delta}{40 M^{2}}\right) .
\end{gathered}
$$

Рассмотрим случайную величину $\xi(x, y):=(f(x)-y)^{2}-\left(f_{\rho}(x)-y\right)^{2}$, где $(x, y) \sim \rho$. Имеем

$$
\mathrm{E} \xi=\mathscr{E}_{\rho}(f), \quad \frac{1}{m} \sum_{i=1}^{m} \xi_{i}=\mathscr{E}_{\rho, \mathbf{z}}(f), \quad|\xi| \leqslant 4 M^{2}
$$

Далее, в силу равенства $\mathrm{E}\left(f(x)-f_{\rho}(x)\right)^{2}=\mathscr{E}_{\rho}(f)$

$\mathrm{D} \xi \leqslant \mathrm{E} \xi^{2}=\mathrm{E}\left[\left(f(x)-f_{\rho}(x)\right)^{2}\left(f(x)+f_{\rho}(x)-2 y\right)^{2}\right] \leqslant 16 M^{2} \mathrm{E}\left(f(x)-f_{\rho}(x)\right)^{2}=16 M^{2} \mathrm{E} \xi$.

Воспользуемся неравенством Берштейна, обозначив $a=\mathrm{E} \xi$ :

$$
\mathrm{P}\left\{\mathrm{E} \xi-\frac{1}{m} \sum_{i=1}^{m} \xi_{i} \geqslant \frac{1}{2}(\mathrm{E} \xi+\delta)\right\} \leqslant \exp \left(-\frac{m((a+\delta) / 2)^{2}}{2\left(16 M^{2} a+(1 / 3) 4 M^{2}(a+\delta) / 2\right)}\right) .
$$

Неравенство (2) следует из (4) и соотношения

$$
\frac{((a+\delta) / 2)^{2}}{2(16 a+(4 / 3)(a+\delta) / 2)} \geqslant \frac{\delta}{40} .
$$

Неравенство (3) доказывается аналогично.

Пусть z таково, что для всех $f \in \mathscr{N}$ выполнено неравенство

$$
\mathscr{E}_{\rho}(f)-\mathscr{E}_{\rho, \mathbf{z}}(f)<\frac{1}{2}\left(\mathscr{E}_{\rho}(f)+\delta\right),
$$

а для $f_{\mathscr{N}}:=\arg \min _{f \in \mathscr{N}} \mathscr{E}(f)$ выполнено еще и

$$
\mathscr{E}_{\rho}(f)-\mathscr{E}_{\rho, \mathbf{z}}(f)>-\frac{1}{2}\left(\mathscr{E}_{\rho}(f)+\delta\right)
$$

Тогда

$$
\mathscr{E}_{\rho}\left(f_{\mathbf{z}, \mathscr{N}}\right) \leqslant \delta+2 \mathscr{E}_{\rho, \mathbf{z}}\left(f_{\mathbf{z}, \mathscr{N}}\right) \leqslant \delta+2 \mathscr{E}_{\rho, \mathbf{z}}\left(f_{\mathscr{N}}\right) \leqslant 2 \delta+3 \mathscr{E}_{\rho}\left(f_{\mathscr{N}}\right) \leqslant 5 \delta
$$

В силу (2) и (3) мера множества таких z больше $1-(N+1) \exp \left(-m \delta /\left(40 M^{2}\right)\right)$, и лемма, тем самым, доказана.

Выведем из леммы теорему А (не заботясь о константах). Из условия теоремы следует оценка

$$
N_{\varepsilon}\left(\Theta, L_{2}(\mu)\right) \leqslant \widehat{N}_{\varepsilon}:=\exp \left(\left(\frac{D}{\varepsilon}\right)^{1 / r}+1\right)
$$


Возьмем оценщик $f_{\mathbf{z}}=f_{\mathbf{z}, \mathscr{N}}$, где $\mathscr{N}$ - минимальная $\sqrt{\varepsilon}$-сеть для $\Theta$ в пространстве $L_{2}(\mu)$. Выберем "критическое" $\varepsilon=\varepsilon^{*}$ из условия $\log \widehat{N}_{\sqrt{\varepsilon^{*}}} \approx c m \varepsilon^{*}$, где $c$ достаточно мало. Очевидно, $\varepsilon^{*} \asymp m^{-2 r /(1+2 r)}$. Теперь применим лемму для выбранного $\mathscr{N}$ и $\delta \geqslant \varepsilon^{*}$; произведение в правой части (1) не превосходит

$$
\exp \left(\log \left(N_{\sqrt{\varepsilon^{*}}}\left(\Theta, L_{2}(\mu)\right)+1\right)-c_{1} m \delta\right) \leqslant \exp \left(-c_{2} m \delta\right),
$$

что и требовалось.

3. Адаптивные оценщики. Пусть заданы произвольное множество индексов $R$ и семейство классов мер $\left\{\mathscr{M}_{r}: r \in R\right\}$.

Пусть для каждого $r$ найдется оценщик $f_{\mathbf{z}}^{r}$ такой, что при всех $\rho \in \mathscr{M}_{r}$ выполняется неравенство

$$
\rho^{m}\left\{\mathbf{z}: \mathscr{E}\left(f_{\mathbf{z}}\right)-\mathscr{E}\left(f_{\rho}\right) \geqslant \varepsilon_{r, m}\right\} \leqslant p_{r, m} .
$$

Здесь $\varepsilon_{r, m}$ и $p_{r, m}$ - некоторые положительные числа, заданные для $r \in R$ и $m \in \mathbb{N}$.

Основное требование заключается в следующем. Для каждого $m \in \mathbb{N}$ в $R$ существует конечное подмножество $R_{m}^{\prime}$ такое, что

$$
\forall r \in R \quad \exists r^{\prime} \in R_{m}^{\prime} \quad \mathscr{M}_{r} \subset \mathscr{M}_{r^{\prime}}, \quad \varepsilon_{r^{\prime}, m} \leqslant \varepsilon_{r, m}^{\prime}, \quad p_{r^{\prime}, m} \leqslant p_{r, m}^{\prime} .
$$

Построим адаптивный оценщик. Для простоты будем считать, что $m$ четно. Разобьем выборку на две части: $\mathbf{z}_{1}=\left(\left(x_{i}, y_{i}\right)\right)_{i=1}^{m / 2}$ и $\mathbf{z}_{2}=\left(\left(x_{i}, y_{i}\right)\right)_{i=m / 2+1}^{m}$. Положим $R^{\prime}:=R_{m / 2}^{\prime}$,

$$
f_{z}:=f_{\mathbf{z}_{1}}^{\widehat{r}}, \quad \widehat{r}:=\arg \min _{r \in R^{\prime}} \mathscr{E}_{\mathbf{z}_{2}}\left(f_{\mathbf{z}_{1}}^{r}\right)=\arg \min _{r \in R^{\prime}} \sum_{i=m / 2+1}^{m}\left(f_{\mathbf{z}_{1}}^{r}\left(x_{i}\right)-y_{i}\right)^{2} .
$$

Теорема 1. Пусть семейство $\left\{\mathscr{M}_{r}\right\}$ и наборы чисел $\varepsilon_{r, m}, \varepsilon_{r, m}^{\prime}, p_{r, m}, p_{r, m}^{\prime}$ таковы, что выполнены условия (5) и (6). Тогда для ощенщика (7) выполнено следующее. Для любой меры $\rho$, лежащей в некотором $\mathscr{M}_{r}$, и любого $\delta_{r, m} \geqslant \varepsilon_{r, m / 2}^{\prime}$ имеет место неравенство

$$
\rho^{m}\left\{\mathbf{z}: \mathscr{E}\left(f_{\mathbf{z}}\right)-\mathscr{E}\left(f_{\rho}\right) \geqslant 5 \delta_{r, m}\right\} \leqslant p_{r, m / 2}^{\prime}+\left(\# R_{m / 2}^{\prime}+1\right) \exp \left(-\frac{m \delta_{r, m}}{80 M^{2}}\right) .
$$

ДоказАтеЛЬСтво. Нам дано, что $\rho \in \mathscr{M}_{r}$. Выберем $r^{\prime}$ в соответствии с (6). Для $\mathbf{z}_{1}$ с вероятностью $\geqslant 1-p_{r^{\prime}, m / 2}$ в силу $\rho \in \mathscr{M}_{r^{\prime}}$ имеем

$$
\mathscr{E}\left(f_{\mathbf{z}_{1}}^{r^{\prime}}\right)-\mathscr{E}\left(f_{\rho}\right)<\varepsilon_{r^{\prime}, m / 2} \leqslant \varepsilon_{r, m / 2}^{\prime}
$$

Зафиксируем $\mathbf{z}_{1}$, для которого это неравенство верно. Применяя основную лемму ко множеству $\mathscr{N}=\left\{f_{\mathbf{z}_{1}}^{r}: r \in R_{m / 2}^{\prime}\right\}$, получим

$$
\rho^{m / 2}\left\{\mathbf{z}_{2}: \mathscr{E}\left(f_{\mathbf{z}}\right)-\mathscr{E}\left(f_{\rho}\right) \geqslant 5 \delta_{r, m}\right\} \leqslant(\# \mathscr{N}+1) \exp \left(\frac{-m \delta_{r, m}}{80 M^{2}}\right) .
$$

Применяя теорему Фубини, получаем утверждение теоремы. 
Теорема В следует из теоремы 1 , если взять

$$
\begin{gathered}
\mathscr{M}_{r}:=\left\{\rho: \rho_{X}=\mu, f_{\rho} \in W_{r}\right\}, \\
\varepsilon_{r, m} \asymp \varepsilon_{r, m}^{\prime} \asymp \delta_{r, m} \asymp A m^{-(2 r) /(1+2 r)}, \\
p_{r, m}=\exp \left(-c A m^{1 /(1+2 r)}\right), \quad p_{r, m}^{\prime}=\exp \left(-c^{\prime} A m^{-2 r /(1+2 r)}\right), \\
R_{m}^{\prime}=\{\alpha\} \cup\left([\alpha, \beta] \cap\left\{\frac{j}{m}: j \in \mathbb{Z}\right\}\right) .
\end{gathered}
$$

Отметим, что условия (5) и (6) построены по аналогии с условиями из [4]. Оценщик строится так же, как и в [4].

4. Мера $\rho_{X}$ неизвестна. Предположим, нам известно лишь, что $f_{\rho} \in \Theta$. В случае фиксированной меры $\rho_{X}=\mu$ оценщик строится по методу наименьших квадратов на подходящей $\sqrt{\varepsilon}$-сети для $\Theta$ в $L_{2}(\mu)$. В рассматриваемом же случае естественно поступить следующим образом:

- зафиксируем $\varepsilon \in(0,1)$;

- разобьем выборку $\mathbf{z}=\left(z_{1}, \ldots, z_{m}\right)$ на две равные части: $\mathbf{z}_{1}$ и $\mathbf{z}_{2}$;

- по $\mathbf{z}_{1}$ построим “эмпирическую $\sqrt{\varepsilon}$-сеть"; точнее, рассмотрим меру

$$
\widehat{\mu}=\frac{1}{m / 2} \sum_{i=1}^{m / 2} \delta_{x_{i}}
$$

и во множестве $\Theta \subset L_{2}(\widehat{\mu})$ построим максимальное по мощности $\sqrt{\varepsilon}$-различимое множество:

$$
\left\|\widehat{f}_{i}-\widehat{f}_{j}\right\|_{L_{2}(\widehat{\mu})}>\sqrt{\varepsilon}, \quad 1 \leqslant i<j \leqslant N
$$

- по $\mathbf{z}_{2}$ и эмпирической сети $\widehat{\mathscr{N}}=\left\{\widehat{f}_{1}, \ldots, \widehat{f}_{N}\right\}$ построим оценщик наименьших квадратов $f_{\mathbf{z}_{2}, \widehat{N}}$;

- окончательно положим $f_{\mathbf{z}}:=f_{\mathbf{z}_{2}, \widehat{N}}$.

Теорема 2. Если $f_{\rho} \in \Theta$, то для построенного оценщика выполняется неравенcmвo

$$
\rho^{n}\left\{\mathbf{z}: \mathscr{E}\left(f_{\mathbf{z}}\right)-\mathscr{E}\left(f_{\rho}\right) \geqslant c_{1} \varepsilon\right\} \leqslant c_{2} \exp \left(c_{3} \mathrm{vc}\left(\Theta, c_{4} \sqrt{\varepsilon}\right) \log \left(\frac{2}{\varepsilon}\right)-c_{5} m \varepsilon\right) .
$$

Здесь и далее через $c, c_{1}, c_{2}, \ldots$ обозначаются абсолютные положительные константы, в разных формулах - разные.

ЗАмечаниЕ. Отметим, что построенный оценщик зависит от $\varepsilon$. Оптимальное $\varepsilon$ следует подбирать из условия $\operatorname{vc}(\Theta, c \sqrt{\varepsilon}) \log (1 / \varepsilon) \asymp m \varepsilon$.

Пусть $\mu$ - мера на $X$. Основой доказательства теоремы 2 служит следующая теорема, позволяющая сравнивать $L_{2}$-норму по мере $\mu$ и по эмпирической мере $\mu_{n}=$ $(1 / n) \sum_{i=1}^{n} \delta_{x_{i}}\left(\right.$ где $\left.x_{i} \sim \mu\right)$. Для краткости обозначим $\|f\|_{n}:=\|f\|_{L_{2}\left(\mu_{n}\right)},\|f\|=$ $\|f\|_{L_{2}(\mu)}$.

Теорема C [5; теорема 11.2]. Пусть $\mathscr{F}$ - класс функиий $X \rightarrow[-M, M]$. Для любого $\eta>0$

$$
\mu^{m}\left\{\mathbf{x}: \exists f \in \mathscr{F}\|f\|-2\|f\|_{n}>\eta\right\} \leqslant 3 \mathrm{E}_{\sqrt{2} \eta / 24}\left(\mathscr{F}, L_{2}\left(\mu_{2 n}\right)\right) \exp \left(-\frac{n \eta^{2}}{288 M^{2}}\right) .
$$


Из следующей теоремы понятно, откуда в нашей задаче возникает комбинаторная размерность.

Tеорема D [6; теорема 1]. Для любого $0<\varepsilon<1$ имеют место неравенства

$$
c_{1} \operatorname{vc}(\mathscr{F}, 2 \varepsilon) \leqslant \sup _{\mu} \log N_{\varepsilon}\left(\mathscr{F}, L_{2}(\mu)\right) \leqslant c_{2} \operatorname{vc}\left(\mathscr{F}, c_{3} \varepsilon\right) \log \left(\frac{2}{\varepsilon}\right),
$$

где точная верхняя грань берется по мерам $\mu$ на $X$ с конечными носителями.

ДокАЗАТЕЛЬСТво теоремы 2. Применим теорему С к классу $\mathscr{F}:=\Theta-\Theta:=$ $\{f-g: f, g \in \Theta\}, \eta=\sqrt{\varepsilon} / 2, n=m / 2$ и мере $\mu=\rho_{X}$. Множитель в правой части (9) оценивается

$$
\mathrm{E} N_{c_{1} \sqrt{\varepsilon}}\left(\mathscr{F}, L_{2}\left(\mu_{m}\right)\right) \leqslant \mathrm{E}\left(N_{c_{2} \sqrt{\varepsilon}}\left(\Theta, L_{2}\left(\mu_{m}\right)\right)\right)^{2} \leqslant \exp \left(c_{3} \operatorname{vc}\left(\Theta, c_{4} \sqrt{\varepsilon}\right) \log \left(\frac{2}{\varepsilon}\right)\right) .
$$

Значит, с большой вероятностью для любых $f, g \in \Theta$

$$
\|f-g\| \leqslant \frac{\sqrt{\varepsilon}}{2}+2\|f-g\|_{m / 2}
$$

Зафиксируем $\mathbf{z}_{1}$, для которого выполнено (11). В силу (11) $\widehat{\mathscr{N}}$ будет $3 \sqrt{\varepsilon}$-сетью для $\Theta$ в $L_{2}\left(\rho_{X}\right)$. Воспользуемся основной леммой для $\mathscr{N}=\widehat{\mathscr{N}}$ и $\delta=(3 \sqrt{\varepsilon})^{2}$ :

$$
\rho^{m / 2}\left\{\mathbf{z}_{2}: \mathscr{E}\left(f_{\mathbf{z}}\right)-\mathscr{E}\left(f_{\rho}\right) \geqslant 45 \varepsilon\right\} \leqslant(\# \widehat{\mathscr{N}}+1) \exp (-c m \varepsilon) .
$$

Далее, очевидно,

$$
\log \# \widehat{N} \leqslant \log N_{\sqrt{\varepsilon} / 2}\left(\Theta, L_{2}\left(\mu_{m}\right)\right) \leqslant c_{1} \operatorname{vc}\left(\Theta, c_{2} \sqrt{\varepsilon}\right) \log \left(\frac{2}{\varepsilon}\right) .
$$

Применяя теорему Фубини, получаем утверждение теоремы.

5. Замечания. Результат теоремы 2 близок к оптимальному. Из нижних оценок [2] следует, что для выпуклого класса $\Theta$ и $\varepsilon>0$, какой бы мы оценщик $f_{\mathbf{z}}$ ни взяли, всегда найдется мера $\rho$ такая, что $f_{\rho} \in \Theta$, но

$$
\rho^{m}\left\{\mathbf{z}: \mathscr{E}\left(f_{\mathbf{z}}\right)-\mathscr{E}\left(f_{\rho}\right) \geqslant \varepsilon\right\} \geqslant \min \left(\frac{1}{2}, \exp \left(c_{1} \operatorname{vc}\left(\Theta, c_{2} \sqrt{\varepsilon}\right)-c_{3} m \varepsilon\right)\right) .
$$

Можно также избавиться и от логарифма в $(8)$ : если $\mathrm{vc}(\Theta, a \varepsilon) \leqslant(1 / 2) \mathrm{vc}(\Theta, \varepsilon)$ для некоторого $a>1$ и всех $\varepsilon>0$, то логарифм в правой части неравенства (10) можно убрать (см. [7; теорема 1.3]). Следовательно, если выполено это условие и класс $\Theta$ выпуклый, построенный нами оценщик оптимален (нужно лишь в доказательстве теоремы 2 использовать неравенство без логарифма).

Тем не менее, остается открытым важный вопрос о построении оценщиков, адаптирующихся к мере $\rho_{X}$. Можно ли построить такой оценщик $f_{\mathbf{z}}$, что для любой меры $\rho$ с $f_{\rho} \in \Theta$ выполнялось бы неравенство

$$
\rho^{m}\left\{\mathbf{z}: \mathscr{E}\left(f_{\mathbf{z}}\right)-\mathscr{E}\left(f_{\rho}\right) \geqslant \varepsilon^{*}\right\} \leqslant c_{1} \exp \left(-c_{2} m \varepsilon^{*}\right),
$$

где $\varepsilon^{*}$ определяется из условия $\log N_{\sqrt{\varepsilon^{*}}}\left(\Theta, L_{2}\left(\rho_{X}\right)\right) \asymp m \varepsilon^{*}$ ?

Построению такого оценщика нашими методами мешает случайная энтропия $N_{\varepsilon}\left(\Theta, L_{2}\left(\mu_{n}\right)\right)$ в теореме С. Применить же теорему 1 невозможно, поскольку классы $\mathscr{M}(\Theta, \mu)=\left\{\rho: f_{\rho} \in \Theta, \rho_{X}=\mu\right\}$ не вложены друг в друга при разных $\mu$. 


\section{СПИСОК ЦИТИРОВАННОЙ ЛИТЕРАТУРЫ}

[1] F. Cucker, S. Smale, "On the mathematical foundations of learning", Bull. Amer. Math. Soc. (N.S.), 39:1 (2002), 1-49.

[2] R. DeVore, G. Kerkyacharian, D. Picard, V. Temlyakov, "Approximation methods for supervised learning", Found. Comput. Math., 6:1 (2006), 3-58.

[3] V.N. Temlyakov, "Approximation in learning theory", Constr. Approx., 27:1 (2008), $33-74$.

[4] С. В. Конягин, Е.Д. Лившиц, "Об адаптивных оценщиках в статистической теории обучения", Теория функиий и нелинейные уравнения в частных производных, Сборник статей. K 70-летию со дня рождения члена-корреспондента РАН Станислава Ивановича Похожаева, Тр. МИАН, 260, МАИК, М., 2008, 193-201.

[5] L. Györfi, M. Kohler, A. Krzyżak, W. Harro, A Distribution-Free Theory of Nonparametric Regression, Springer Ser. Statist., Springer-Verlag, New York, NY, 2002.

[6] S. Mendelson, R. Vershynin, "Entropy and the combinatorial dimension", Invent. Math., 152:1 (2003), 37-55.

[7] M. Rudelson, R. Vershynin, "Combinatorics of random processes and sections of convex bodies", Ann. of Math. (2), 164:2 (2006), 603-648.

Ю. В. Малыхин

Математический институт им. В. А. Стеклова РАН

E-mail: jura05@narod.su
Поступило

20.11.2008

Исправленный вариант

28.02.2009 